\title{
Numerical solution of the one-dimensional fractional convection diffusion equations based on Chebyshev operational matrix
}

\author{
Jiaquan Xie*, Qingxue Huang and Xia Yang
}

${ }^{*}$ Correspondence: xja371195982@163.com College of Mechanical Engineering, Taiyuan University of Science and Technology, Taiyuan 030024, Shanxi, China

\begin{abstract}
In this paper, we are concerned with nonlinear one-dimensional fractional convection diffusion equations. An effective approach based on Chebyshev operational matrix is constructed to obtain the numerical solution of fractional convection diffusion equations with variable coefficients. The principal characteristic of the approach is the new orthogonal functions based on Chebyshev polynomials to the fractional calculus. The corresponding fractional differential operational matrix is derived. Then the matrix with the Tau method is utilized to transform the solution of this problem into the solution of a system of linear algebraic equations. By solving the linear algebraic equations, the numerical solution is obtained. The approach is tested via examples. It is shown that the proposed algorithm yields better results. Finally, error analysis shows that the algorithm is convergent.
\end{abstract}

Keywords: Chebyshev operational matrix, One-dimensional fractional convection diffusion equations, Numerical solution, Variable coefficients, Tau method

\section{Background}

Convection diffusion equations are regarded as a kind of basic equations of motion, which have been applied in describing water flow movement (Hu et al. 2016; Colla et al. 2015; Su 2014), material transport and diffusion (Liu et al. 2016; Calo et al. 2015; Karalashvili et al. 2015; Fang and Deng 2014). Convection diffusion equations are widely used in water conservancy project ( $\mathrm{Hu}$ et al. 2016; Su 2014), environmental engineering and aviation (Hernandez et al. 1995), Marine (Farahani et al. 2015), chemical (Colla et al. 2015; Diehl 2015), metallurgy (Zaib and Shafile 2014), so the study of numerical solutions of convection diffusion equations has important theoretical and practical significance.

There are many numerical methods for solving convection diffusion problems, such as finite difference methods (Kaya 2015), finite element methods (He et al. 2015; Mudunuru and Nakshatrala 2016; Wu et al. 2013), wavelet methods (Zhou and Xu 2014, 2016; Chen et al. 2010; EI-Gamel 2006), polynomials methods (Li et al. 2016), iterative methods (Das and Mehrmann 2015; Das 2015; Das and Natesan 2014). Operational matrix 
methods are new on this area which have made great success presently. Especially in recent 20 years, the articles about operational matrix methods are springing up. In Ref. Abbasbandy et al. (2015), the authors proposed the operational matrix method of fractional-order Legendre functions for solving time-fractional convection-diffusion equations.

In the following we mention some real-world applications of convection diffusion equations. In this paper, we take an example of a diffusion kinetic model, the flow rate is $\boldsymbol{u}=u(x, t)$, dye concentration is $c(x, t)$ and diffusion flux of dyes is $\boldsymbol{q}=q(x, t)$. We also set up a variety of biological, chemical and other factors to control the production rate of dye (Production of unit volume per unit time) $F_{c}$. In the flow field, a system (its volume can be arbitrary), as shown in Fig. 1, is obtained. The space occupied by the system during the flow of $V(t)$ is controlled at the time $t$.

According to the model, and the related initial-boundary conditions, we obtain the one-dimensional convection diffusion equations (Chen and Jin (2007)).

$$
\begin{cases}\frac{\partial c}{\partial t}+u \frac{\partial c}{\partial x}=D \frac{\partial^{2} c}{\partial x^{2}}, & t>0,0<x<l \\ c(x, 0)=0, & 0<x<l \\ c(0, t)=C_{0}, & c(l, t)=0, t>0\end{cases}
$$

In this paper, a numerical approach based on Chebyshev operational matrix is proposed for solving one-dimensional fractional convection diffusion equations with variable coefficients of the following form:

$$
\begin{cases}\frac{\partial u}{\partial t}+a(x) \frac{\partial u}{\partial x}=b(x) \frac{\partial^{v} u}{\partial x^{v}}+g(x, t), & 0<x<1, t>0, \\ u(x, 0)=h_{1}(x), & 0<x<1, \\ u(0, t)=g_{1}(t), u(1, t)=g_{2}(t), & t>0,\end{cases}
$$

the parameter $v$ refers to the fractional order of spatial derivative with $1<v \leq 2$. In the proposed method, the operational matrices of fractional-order are employed to obtain the numerical solutions of Eq. (2).

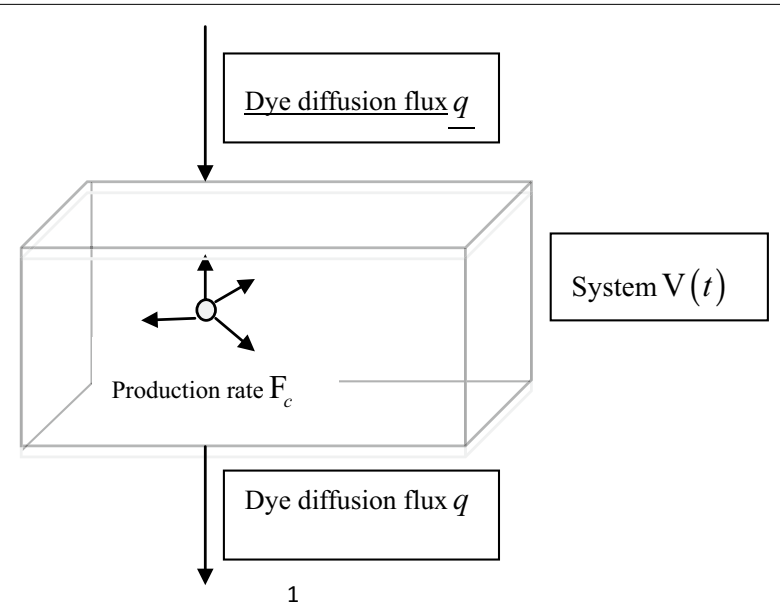

Fig. 1 The schematic diagram of dye diffusion in the system 
The paper is organized as follows: In "Preliminaries and notations" section, some basic definitions and mathematical preliminaries of fractional calculus are introduced. The fractional differential operational matrix is given in "The fractional derivative operational matrix $P^{(v)}$ " section. We mainly illustrate the proposed method in "Description of the proposed method" section. In "Error analysis" section, the convergence of the proposed approach is proved. In "Numerical simulation" section, the proposed approach is applied to test several numerical examples. Finally, a conclusion is given in "Conclusion" section.

\section{Preliminaries and notations}

\section{The basic definitions of fractional integral and differential operator}

Definition 1 The Riemann-Liouville fractional integral operator $I^{v}$ of order $v$ is defined as

$$
\left(I^{v} f\right)(t)= \begin{cases}\frac{1}{\Gamma(v)} \int_{0}^{t}(t-\tau)^{\nu-1} f(\tau) d \tau, & v>0 \\ f(t), & v=0\end{cases}
$$

Definition 2 The Riemann-Liouville fractional differential operator $D^{v}$ of order $v$ is defined as

$$
D^{v} f(t)= \begin{cases}\frac{1}{\Gamma(m-v)} \frac{\mathrm{d}^{m}}{\mathrm{~d} t^{m}} \int_{0}^{t} \frac{f(s)}{(t-s)^{v-m+1}} d s, & v>0, m-1 \leq v<m ; \\ \frac{\mathrm{d}^{m} f(t)}{\mathrm{d} t^{m}}, & v=m .\end{cases}
$$

Definition 3 The Caputo fractional differential operator is defined as

$$
D^{v} f(t)= \begin{cases}\frac{1}{\Gamma(m-v)} \int_{0}^{t} \frac{f^{(m)}(\tau)}{(t-\tau)^{v-m+1}} d \tau, & m-1 \leq v<m ; \\ \frac{\mathrm{d}^{m} f(t)}{\mathrm{d} t^{m}}, & v=m .\end{cases}
$$

For the Caputo derivative, we have

$$
D^{v} t^{\gamma}= \begin{cases}0, & \text { for } \gamma \in N_{0} \text { and } \gamma<\lceil\nu\rceil ; \\ \frac{\Gamma(\gamma+1)}{\Gamma(\gamma+1-v)} t^{\gamma-\nu}, & \text { for } \gamma \in N_{0} \text { and } \gamma \geq\lceil\nu\rceil \text { or } \gamma \notin N_{0} \text { and } \gamma>\lfloor v\rfloor .\end{cases}
$$

\section{Properties of the Chebyshev polynomials}

The well-known Chebyshev polynomials are defined on the interval $(-1,1)$ and can be determined with the aid of the following recurrence formula (Doha et al. 2011):

$$
T_{i+1}(t)=2 t T_{i}(t)-T_{i-1}(t), \quad i=1,2, \ldots
$$

where $T_{0}(t)=1$ and $T_{1}(t)=t$. In order to use these polynomials on the interval $x \in(0,1)$, we define the Chebyshev polynomials by introducing the change of variable $t=2 x-1$. Let the Chebyshev polynomials $T_{i}(t)=2 x-1$ are denoted by $T_{i}(x)$, then $T_{i}(x)$ can be obtained as follows:

$$
T_{i+1}(x)=2(2 x-1) T_{i}(x)-T_{i-1}(x), \quad i=1,2, \ldots
$$

where $T_{0}(x)=1$ and $T_{1}(x)=2 x-1$. The analytic form of the Chebyshev polynomials $T_{i}(x)$ of degree $i$ is given by 


$$
T_{i}(x)=i \sum_{k=0}^{i}(-1)^{i-k} \frac{(i+k-1) ! 2^{2 k}}{(i-k) !(2 k) !} x^{k}
$$

where $T_{i}(0)=(-1)^{i}$ and $T_{i}(1)=1$.

The orthogonally condition is

$$
\int_{0}^{1} T_{j}(x) T_{k}(x) w(x) d x=h_{k}
$$

where the weight function $w(x)=\frac{1}{\sqrt{x-x^{2}}}$ and $h_{k}= \begin{cases}\frac{b_{k}}{2} \pi, & k=j, \quad b_{0}=2, b_{k}=1, k \geq 1 . \\ 0, & k \neq j,\end{cases}$

\section{Function approximation}

Suppose $u(x) \in L^{2}(0,1)$, it may be expressed in terms of the Chebyshev polynomials as

$$
u(x)=\sum_{i=0}^{\infty} c_{i} T_{i}(x)
$$

where the coefficients $c_{i}$ is given by

$$
c_{i}=\frac{1}{h_{i}} \int_{0}^{1} u(x) T_{i}(x) w(x) d x, \quad i=0,1,2, \ldots
$$

If we consider truncated series in Eq. (10), then we have:

$$
u(x) \simeq u_{M}(x)=\sum_{i=0}^{M} c_{i} T_{i}(x)=C^{T} \boldsymbol{\Phi}(x)
$$

where

$$
\boldsymbol{C}=\left[c_{0}, c_{1}, \ldots, c_{M}\right]^{T} ; \quad \boldsymbol{\Phi}(x)=\left[T_{0}(x), T_{1}(x), \ldots, T_{M}(x)\right]^{T} .
$$

Then the derivative of vector $\Phi$ can be expressed by

$$
\frac{\mathrm{d} \boldsymbol{\Phi}(x)}{\mathrm{d} x}=\boldsymbol{P}^{(1)} \boldsymbol{\Phi}(x),
$$

where $P^{(1)}$ is the $(M+1) \times(M+1)$ operational matrix of derivative given by

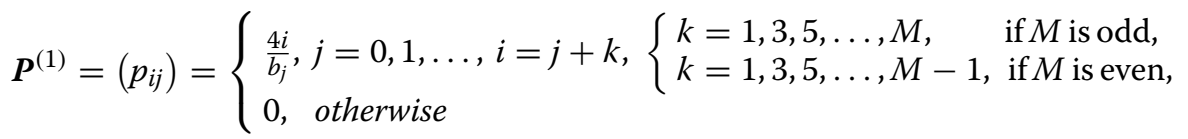

For example for even $M$, we have

$$
\boldsymbol{P}^{(1)}=2 \times\left(\begin{array}{cccccccc}
0 & 0 & 0 & 0 & 0 & \cdots & 0 & 0 \\
1 & 0 & 0 & 0 & 0 & \cdots & 0 & 0 \\
0 & 4 & 0 & 0 & 0 & \cdots & 0 & 0 \\
3 & 0 & 6 & 0 & 0 & \cdots & 0 & 0 \\
0 & 8 & 0 & 8 & 0 & \cdots & 0 & 0 \\
5 & 0 & 10 & 0 & 10 & \cdots & 0 & 0 \\
\vdots & \vdots & \vdots & \vdots & \vdots & \cdots & \vdots & \vdots \\
M-1 & 0 & 2 M-2 & 0 & 2 M-2 & \cdots & 0 & 0 \\
0 & 2 M & 0 & 2 M & 0 & \cdots & 2 M & 0
\end{array}\right)
$$


Based on the function approximation theory which the solution function is expressed as orthogonal polynomials. For arbitrary function $u(x, t) \in L^{2}((0,1) \times(0,1))$, it can be expanded as the following way

$$
u(x, t)=\sum_{i=0}^{\infty} \sum_{j=0}^{\infty} u_{i j} T_{i}(x) T_{j}(t)
$$

where

$$
u_{i j}=\frac{1}{h_{i} h_{j}} \int_{0}^{1} \int_{0}^{1} u(x, t) T_{i}(x) T_{j}(t) w(x) w(t) d x d t, \quad i, j=0,1,2, \ldots
$$

If we consider truncated series in Eq. (16), then we have:

$$
u(x, t) \approx \sum_{i=0}^{M} \sum_{j=0}^{N} u_{i j} T_{i}(x) T_{j}(t)=\boldsymbol{\Phi}(x)^{T} \boldsymbol{U} \boldsymbol{\Phi}(t),
$$

where

$$
\boldsymbol{\Phi}(x)=\left[T_{0}(x), T_{1}(x), \ldots, T_{M}(x)\right]^{T} ; \boldsymbol{\Phi}(t)=\left[T_{0}(t), T_{1}(t), \ldots, T_{N}(t)\right]^{T} ; \boldsymbol{U}=\left\{u_{i j}\right\}_{i, j=0}^{M, N} .
$$

In this paper, we use the Tau method (Bhrawy et al. 2011; Dehghan and Saadatmandi 2006) to compute the coefficients $u_{i j}$.

\section{The fractional derivative operational matrix $P^{(v)}$}

The main objective of this section is to prove the following theorem for the fractional derivatives of the Chebyshev polynomials.

Lemma 1 Let $T_{i}(x)$ be a Chebyshev polynomial; then (Doha et al. 2011)

$$
D^{\nu} T_{i}(x)=0, \quad i=0,1,2, \ldots,\lceil\nu\rceil-1, \quad \nu>0 .
$$

Proof This Lemma can be easily proved by making use of relation (5) and (7).

Theorem 1 Let $\boldsymbol{\Phi}(x)$ be the Chebyshev vector defined in Eq. (12) and suppose v >0; then

$$
D^{v} \boldsymbol{\Phi}(x) \simeq \boldsymbol{P}^{(v)} \boldsymbol{\Phi}(x),
$$

where $P^{(v)}$ is the $(M+1) \times(M+1)$ differential operational matrix of order $v$ in the Caputo sense and it is defined as follows: 


$$
\boldsymbol{P}^{(v)}=\left(\begin{array}{ccccc}
0 & 0 & 0 & \cdots & 0 \\
\vdots & \vdots & \vdots & \cdots & \vdots \\
0 & 0 & 0 & \cdots & 0 \\
S_{v}(\lceil\nu\rceil, 0) & S_{v}(\lceil\nu\rceil, 1) & S_{v}(\lceil\nu\rceil, 2) & \cdots & S_{v}(\lceil\nu\rceil, M) \\
\vdots & \vdots & \vdots & \cdots & \vdots \\
S_{v}(i, 0) & S_{v}(i, 1) & S_{v}(i, 2) & \cdots & S_{v}(i, M) \\
\vdots & \vdots & \vdots & \cdots & \vdots \\
S_{v}(M, 0) & S_{v}(M, 1) & S_{v}(M, 2) & \cdots & S_{v}(M, M)
\end{array}\right)
$$

where

$$
\begin{aligned}
S_{\nu}(i, j)= & \sum_{\substack{k=\lceil\nu\rceil \\
i}} \frac{(-1)^{i-k} 2 i(i+k-1) ! \Gamma\left(k-v+\frac{1}{2}\right)}{b_{j} \Gamma\left(k+\frac{1}{2}\right)(i-k) ! \Gamma(k-v-j+1) \Gamma(k-v+j+1)} . \\
& i=\lceil\nu\rceil,\lceil\nu\rceil+1, \ldots, M .
\end{aligned}
$$

Proof The analytical form of the Chebyshev polynomials $T_{i}(x)$ of degree $i$ is given by Eq. (8), using Eqs. (6) and (8) we have

$$
\begin{aligned}
D^{\nu} T_{i}(x) & =i \sum_{k=0}^{i}(-1)^{i-k} \frac{(i+k-1) ! 2^{2 k}}{(i-k) !(2 k) !} D^{v} x^{k} \\
& =i \sum_{k=0}^{i}(-1)^{i-k} \frac{(i+k-1) ! 2^{2 k} k !}{(i-k) !(2 k) ! \Gamma(k+v+1)} x^{k-v}, \quad i=\lceil\nu\rceil,\lceil\nu\rceil+1, \ldots, M .
\end{aligned}
$$

Now, approximate $x^{k-v}$ by $(M+1)$ terms of the Chebyshev series, we have

$$
x^{k-v}=\sum_{j=0}^{M-1} c_{k j} T_{j}(x)
$$

where $c_{k j}$ is given from Eq. (11) with $u(x)=x^{k-v}$, and

$$
c_{k j}= \begin{cases}\frac{1}{\sqrt{\pi}} \frac{\Gamma\left(k-v+\frac{1}{2}\right)}{\Gamma(k-v+1)}, & j=0, \\ \frac{j}{\sqrt{\pi}} \sum_{r=0}^{j}(-1)^{j-r} \frac{(j+r-1) ! 2^{2 r+1} \Gamma\left(k+r-v+\frac{1}{2}\right)}{(j-r) !(2 r) ! \Gamma(k+r-v+1)}, & j=1,2, \ldots M .\end{cases}
$$

Employing Eqs. (22)-(24) we get

$$
D^{\nu} T_{i}(x)=\sum_{j=0}^{M} S_{\nu}(i, j) T_{j}(x), \quad i=\lceil\nu\rceil,\lceil\nu\rceil+1, \ldots M .
$$

where $S_{v}(i, j)=\sum_{k=\lceil v\rceil}^{i} \theta_{i j k}$, and 


$$
\theta_{i j k}= \begin{cases}\frac{i(-1)^{i-k}(i+k-1) ! 2^{2 k} k ! \Gamma\left(k-v+\frac{1}{2}\right)}{(i-k) !(2 k) ! \sqrt{\pi}(\Gamma(k-v+1))^{2}}, & j=0, \\ \frac{(-1)^{i-k} i j(i+k-1) ! 2^{2 k+1} k !}{(i-k) !(2 k) ! \Gamma(k-v+1) \sqrt{\pi}} & \\ \times \sum_{r=0}^{j} \frac{(-1)^{j-r}(j+r-1) ! 2^{2 r} \Gamma\left(k+r-v+\frac{1}{2}\right)}{(j-r) !(2 r) ! \Gamma(k+r-v+1)}, & j=1,2, \ldots M .\end{cases}
$$

After some lengthy manipulation, $\theta_{i, j, k}$ may be put in the form

$$
\theta_{i j k}=\frac{(-1)^{i-k} 2 i(i+k-1) ! \Gamma\left(k-v+\frac{1}{2}\right)}{b_{j} \Gamma\left(k+\frac{1}{2}\right)(i-k) ! \Gamma(k-v-j+1) \Gamma(k-v+j+1)}, \quad j=0,1, \ldots M .
$$

where $b_{0}=2, b_{j}=1, j \geq 1$.

Accordingly, Eq. (21) can be written in a vector form as follows:

$D^{v} T_{i}(x) \simeq\left[S_{v}(i, 0), S_{v}(i, 1), S_{v}(i, 2), \ldots S_{v}(i, M)\right] \Phi(x), \quad i=\lceil\nu\rceil,\lceil v\rceil+1, \ldots, M$.

Also, according to Lemma 1, we can write

$$
D^{\nu} T_{i}(x) \simeq[0,0,0, \ldots, 0] \boldsymbol{\Phi}(x), \quad i=0,1, \ldots,\lceil\nu\rceil-1 .
$$

A combination of Eqs. (27) and (28) leads to the desired result.

\section{Description of the proposed method}

In the section, we will use the Chebyshev polynomials operational matrix of fractional derivative to obtain the numerical solutions of one-dimensional fractional convection diffusion equations with variable coefficients.

Here, for simplicity we consider the convection diffusion equations of the following form:

$$
\frac{\partial u}{\partial t}+a(x) \frac{\partial u}{\partial x}=b(x) \frac{\partial^{v} u}{\partial x^{v}}+f(x, t), \quad 1<v \leq 2 .
$$

where $\partial^{v} / \partial x^{v}$ denote fractional derivatives in the Caputo's sense, with the initial-boundary conditions

$$
\begin{aligned}
& u(x, 0)=h_{1}(x), \quad 0<x<1, \\
& u(0, t)=g_{1}(x), u(1, t)=g_{2}(t), \quad t>0 .
\end{aligned}
$$

In order to use the Chebyshev polynomials, we first approximate

$$
u(x, t) \approx \boldsymbol{\Phi}^{\mathrm{T}}(x) \boldsymbol{U} \boldsymbol{\Phi}(t),
$$

where $\boldsymbol{U}=\left[u_{i j}\right]_{(M+1) \times(N+1)}$ is an unknown matrix.

The following is the product of two vectors based on Chebyshev operational matrix method. Let (Bhrawy et al. 2015)

$$
\boldsymbol{\Phi}(x) \boldsymbol{\Phi}^{T}(x) \boldsymbol{C} \approx \tilde{\boldsymbol{Q}}^{T} \boldsymbol{\Phi}(x),
$$


where $\tilde{\boldsymbol{Q}}$ is the $(M+1) \times(M+1)$ operational matrix with the element $\tilde{\boldsymbol{q}}_{k j}$. In virtue of Eq. (12) and above relation, enable us to write

$$
\sum_{i=0}^{M} c_{i} T_{i}(x) T_{j}(x)=\sum_{i=0}^{M} \tilde{\boldsymbol{q}}_{i j} T_{i}(x), \quad j=0,1,2, \ldots, M .
$$

Multiplying both sides of the above equation by $T_{k}(x) \omega(x), k=0,1, \ldots, M$ and integrating the result from 0 to 1 , we obtain

$$
\sum_{i=0}^{M} c_{i} \int_{0}^{1} T_{i}(x) T_{j}(x) T_{k}(x) \omega(x) d x=\tilde{\boldsymbol{q}}_{k j} \int_{0}^{1} T_{k}(x) T_{k}(x) \omega(x) d x
$$

Equation (34) yields

$$
\tilde{\boldsymbol{q}}_{k j}=\frac{1}{h_{k}} \sum_{i=0}^{M}\left(q_{i} \int_{0}^{1} T_{i}(x) T_{j}(x) T_{k}(x) \omega(x)\right), \quad k, j=0,1, \ldots, M .
$$

Now, using Eqs. (12), (14), (21) and (32), we obtain

$$
\begin{aligned}
& \frac{\partial u}{\partial t} \approx \boldsymbol{\Phi}^{T}(x) \boldsymbol{U} \boldsymbol{P}^{(1)} \boldsymbol{\Phi}(t), \\
& a(x) \frac{\partial u}{\partial x} \approx A^{T} \boldsymbol{\Phi}(x) \boldsymbol{\Phi}^{T}(x)\left(\boldsymbol{P}^{(1)}\right)^{T} \boldsymbol{U} \boldsymbol{\Phi}(t) \approx \boldsymbol{\Phi}^{T}(x) \tilde{\boldsymbol{A}}^{T}\left(\boldsymbol{P}^{(1)}\right)^{T} \boldsymbol{U} \boldsymbol{\Phi}(t),
\end{aligned}
$$

and

$$
b(x) \frac{\partial^{v} u}{\partial x^{v}} \approx \boldsymbol{B}^{T} \boldsymbol{\Phi}(x) \boldsymbol{\Phi}^{T}(x)\left(\boldsymbol{P}^{v}\right)^{T} \boldsymbol{U} \boldsymbol{\Phi}(t) \approx \boldsymbol{\Phi}^{T}(x) \tilde{\boldsymbol{B}}^{T}\left(\boldsymbol{P}^{v}\right)^{T} \boldsymbol{U} \boldsymbol{\Phi}(t),
$$

Also, using Eq. (18), the function $g(x, t)$ in Eq. (29) can be approximated as

$$
f(x, t) \approx \boldsymbol{\Phi}^{T}(x) \boldsymbol{F} \boldsymbol{\Phi}(t),
$$

where $\boldsymbol{F}=\left[f_{i j}\right]$ is a $(M+1) \times(N+1)$ known matrix. Substituting Eqs. (31)-(39) in Eq. (29) yields

$$
\boldsymbol{\Phi}^{T}(x) \boldsymbol{U} \boldsymbol{P}^{(1)} \boldsymbol{\Phi}(t)+\boldsymbol{\Phi}^{T}(x) \tilde{\boldsymbol{A}}^{T}\left(\boldsymbol{P}^{(1)}\right)^{T} \boldsymbol{U} \boldsymbol{\Phi}(t) \approx \boldsymbol{\Phi}^{T}(x) \tilde{\boldsymbol{B}}^{T}\left(\boldsymbol{P}^{v}\right)^{T} \boldsymbol{U} \boldsymbol{\Phi}(t)+\boldsymbol{\Phi}^{T}(x) \boldsymbol{G} \boldsymbol{\Phi}(t)
$$

The entries of vector $\boldsymbol{\Phi}(x)$ and $\boldsymbol{\Phi}(t)$ in Eq. (40) are independent, so we get

$$
H=\boldsymbol{U} \boldsymbol{P}^{(1)}+\tilde{\boldsymbol{A}}^{T}\left(\boldsymbol{P}^{(1)}\right)^{T} \boldsymbol{U}-\tilde{\boldsymbol{B}}^{T}\left(\boldsymbol{P}^{v}\right)^{T} \boldsymbol{U}-\boldsymbol{F} \approx 0
$$

Here, we choose $M N-N$ equations of Eq. (41) as

$$
H_{i j} \approx 0, \quad(i=2,3, \ldots, M, j=1,2, \ldots, N)
$$

We can also approximate the function $h_{1}(x), g_{1}(t)$ and $g_{2}(t)$ as 


$$
h_{1}(x) \approx \boldsymbol{\Phi}^{T}(x) \boldsymbol{H}_{1}, g_{1}(t) \approx \boldsymbol{G}_{1}^{T} \boldsymbol{\Phi}(t), g_{2}(t) \approx \boldsymbol{G}_{2}^{T} \boldsymbol{\Phi}(t)
$$

where $\boldsymbol{H}_{1}, \boldsymbol{G}_{1}, \boldsymbol{G}_{2}$ are known vectors.

Applying Eqs. (31) and (44) in the boundary conditions Eq. (30), we get

$$
\begin{aligned}
& \boldsymbol{\Phi}^{T}(x) \boldsymbol{U} \boldsymbol{\Phi}(0) \approx \boldsymbol{\Phi}^{T}(x) \boldsymbol{H}_{1}, \\
& \boldsymbol{\Phi}^{T}(0) \boldsymbol{U} \boldsymbol{\Phi}(t) \approx \boldsymbol{G}_{1}^{T} \boldsymbol{\Phi}(t), \boldsymbol{\Phi}^{T}(1) \boldsymbol{U} \boldsymbol{\Phi}(t) \approx \boldsymbol{G}_{2}^{T} \boldsymbol{\Phi}(t),
\end{aligned}
$$

The entries of vector $\boldsymbol{\Phi}(x)$ and $\boldsymbol{\Phi}(t)$ are independent, so from Eq. (44) we can obtain

$$
\Lambda_{1}=\boldsymbol{U} \boldsymbol{\Phi}(0)-\boldsymbol{H}_{1} \approx 0, \Lambda_{2}=\boldsymbol{\Phi}^{T}(0) \boldsymbol{U}-\boldsymbol{G}_{1}^{T} \approx 0, \Lambda_{3}=\boldsymbol{\Phi}^{T}(1) \boldsymbol{U}-\boldsymbol{G}_{2}^{T} \approx 0,
$$

By choosing the $(M-1)$ equations of $\Lambda_{1}=0$ and $(N+1)$ equations of $\Lambda_{j}=0 \quad(j=2,3)$, we get $2 N+M+1$ equations, i.e.

$$
\begin{gathered}
\Lambda_{j i} \approx 0, \quad j=1 \quad i=1, \ldots, M-1 . \\
\Lambda_{j i} \approx 0, \quad j=2,3, \quad i=0,1, \ldots, N .
\end{gathered}
$$

Equation (42) together with Eq. (46) gives $(M+1)(N+1)$ equations, which can be solved for $u_{i j},(i=0,1, \ldots, M, j=0,1, \ldots, N)$. So the unknown function $u(x, t)$ can be find out.

\section{Error analysis}

In real problems, we often tend to solve some equations with unknown exact solutions. Hence, when we apply our method to these kinds of problems, it is necessary to introduce a process for estimating the error function (Chen et al. 2014).

We consider $e_{n}(x, t)=u(x, t)-u_{m n}(x, t)$ as the error function of the approximate solution $u_{m n}(x, t)$ for $u(x, t)$, where $u(x, t)$ is the exact solution of Eq. (2)

Therefore, $u_{n}(x, t)$ satisfies the following problem

$$
\frac{\partial u_{m n}(x, t)}{\partial t}+a(x) \frac{\partial u_{m n}(x, t)}{\partial x}=b(x) \frac{\partial^{v} u_{m n}(x, t)}{\partial x^{v}}+f(x, t)+R_{m n}(x, t)
$$

The perturbation term $R_{m n}(x, t)$ can be obtained by substituting the estimated solution $u_{m n}(x, t)$ into the equations:

$$
R_{m n}(x, t)=\frac{\partial u_{m n}(x, t)}{\partial t}+a(x) \frac{\partial u_{m n}(x, t)}{\partial x}-b(x) \frac{\partial^{v} u_{m n}(x, t)}{\partial x^{v}}-f(x, t)
$$

Subtracting Eq. (47) from Eq. (2), we get the following equations:

$$
\frac{\partial e_{m n}(x, t)}{\partial t}+a(x) \frac{\partial e_{m n}(x, t)}{\partial x}-b(x) \frac{\partial^{v} e_{m n}(x, t)}{\partial x^{v}}=-R_{m n}(x, t)
$$

Obviously the above equation is one-dimensional fractional convection diffusion equation in which the error function $e_{m n}(x, t)$, is the unknown function. We can easily apply our method to the above equation to find an approximation of the error function $e_{m n}^{\prime}(x, t)$. 


\section{Numerical simulation}

In this section, we apply the proposed algorithm in the previous section to obtain numerical solutions of some convection diffusion equations with variable coefficients.

Example 1 Consider the two-dimensional fractional convection diffusion equations with homogeneous initial-boundary conditions

$$
\frac{\partial u(x, t)}{\partial t}+2 \frac{\partial u(x, t)}{\partial x}=\frac{\partial^{1.5} u(x, t)}{\partial x^{1.5}}+f(x, t) \quad 0<x<1, t>0
$$

where $f(x, t)=x(x-1)(2 t-1)+2 t(t-1)(2 x-1)-4 \sqrt{x} t(t-1) / \sqrt{\pi}$, and the initial-boundary conditions:

$$
\begin{aligned}
& u(x, 0)=0 \\
& u(0, t)=u(1, t)=0, \quad t>0 .
\end{aligned}
$$

The exact solution of this problem is $u(x, t)=x t(x-1)(t-1)$. The graphs of numerical solution for $M=N=4$ is shown in Fig. 2. Absolute error between the numerical and analytical solutions is shown in Fig. 3. The graphs of analytical and approximate solutions for some nodes in $(0,1) \times(0,1)$ are presented in Fig. 4 . Absolute error between the numerical and analytical solutions are also shown at different times in Fig. 5.

Figures 2 and 4 show that the numerical solutions are very close to the analytical solutions. Figures 3 and 5 show that the proposed algorithm has a high convergence precision.

Example 2 Consider the fractional convection diffusion equations with variable coefficients

$$
\frac{\partial u}{\partial t}+a(x) \frac{\partial u}{\partial x}=b(x) \frac{\partial^{2} u}{\partial x^{2}}+f(x, t) ; \quad 0<x<1, t>0
$$

with the initial-boundary conditions:

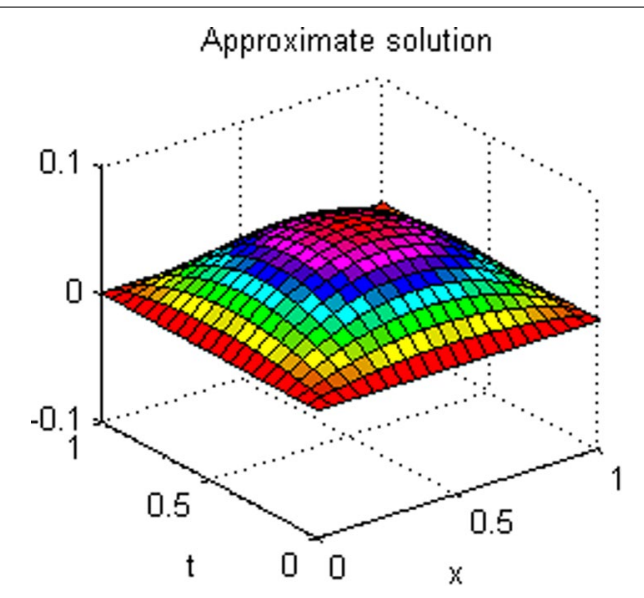

Fig. 2 Approximate solution of Example 1 


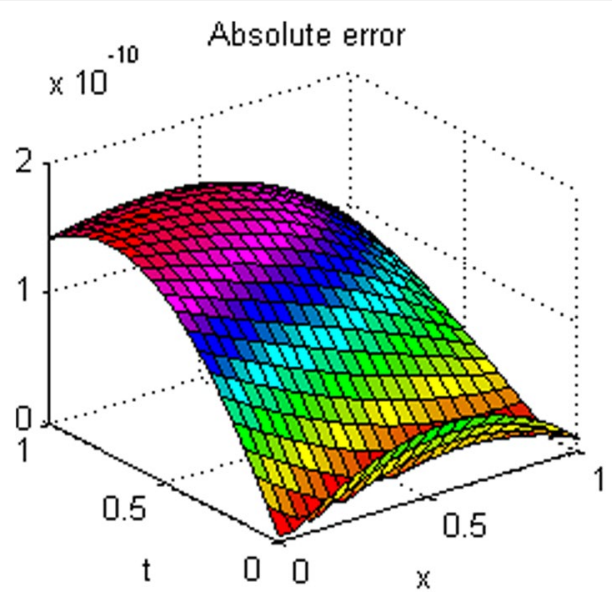

Fig. 3 Absolute error of Example 1

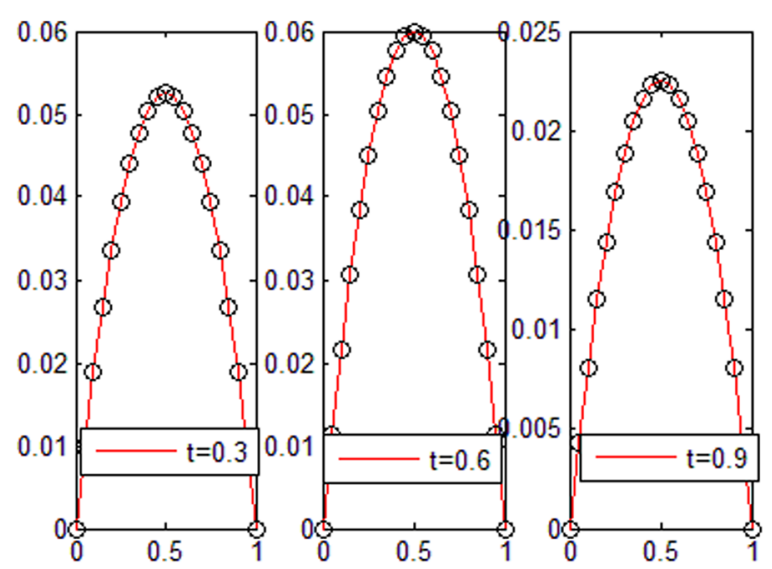

Fig. 4 Numerical and exact solution in different values of $t$ for Example 1

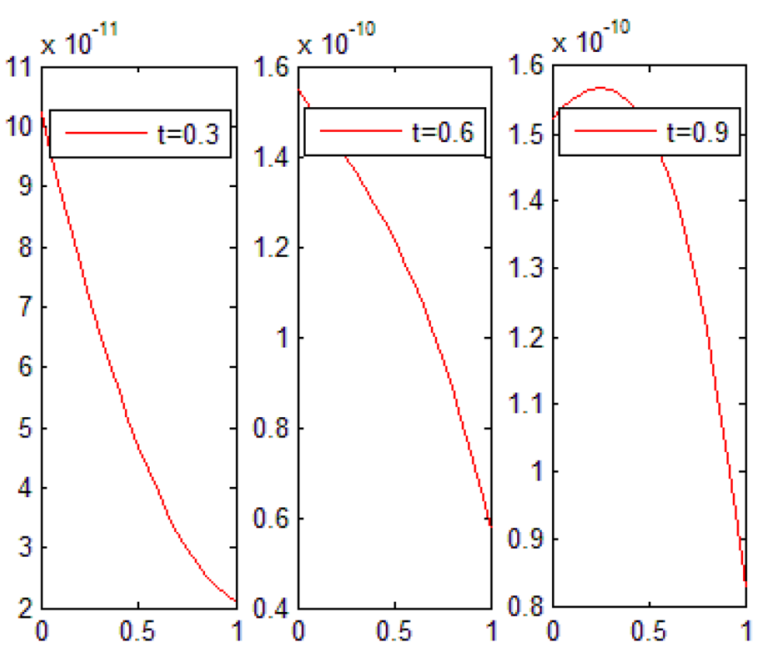

Fig. 5 Absolute error in different values of $t$ for Example 1 


$$
\begin{aligned}
& u(x, 0)=0, \\
& u(0, t)=0, u(1, t)=\sinh (t), \quad t>0
\end{aligned}
$$

where $a(x)=\frac{x}{3}, \quad b(x)=\frac{x^{2}}{6} ; f(x, t)=x^{3} \cosh (t)$. The exact solution of this problem is $u(x, t)=x^{3} \sinh (t)$. The graph of the numerical solution for $M=N=7$ is shown in Fig. 6 . Absolute error between the numerical and analytical solutions is shown in Fig. 7. The graphs of analytical and numerical solution for different $M$ and $N$ in some nodes are shown in Fig. 8. Absolute error between the numerical and analytical solution are also shown at different times in Fig. 9.

From Figs. 6 and 8, we can conclude that the numerical solutions converge to the exact solutions very well. Figures 7 and 9 show that the proposed algorithm can get a high

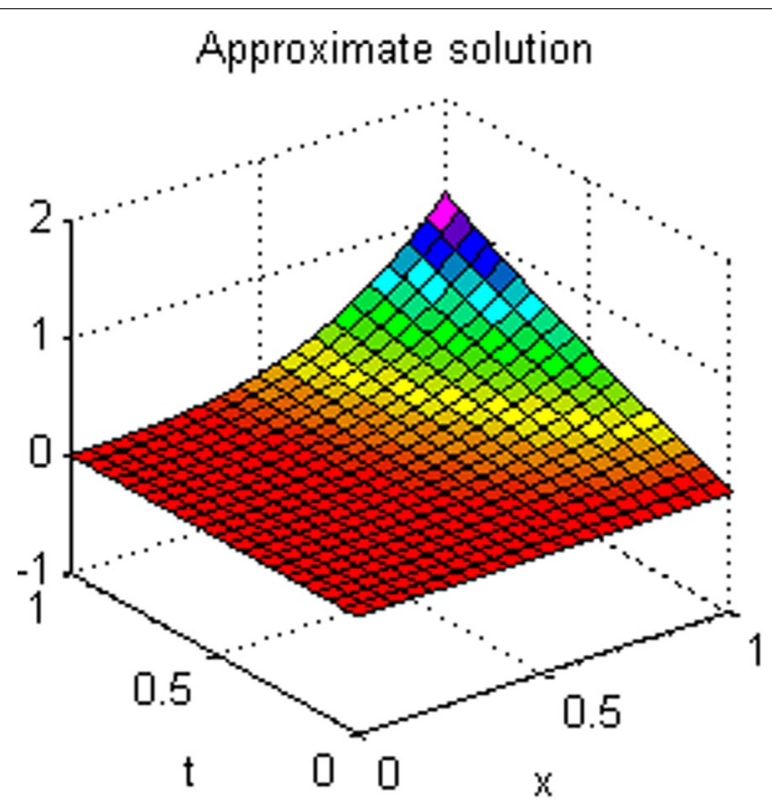

Fig. 6 Approximate solution of Example 2

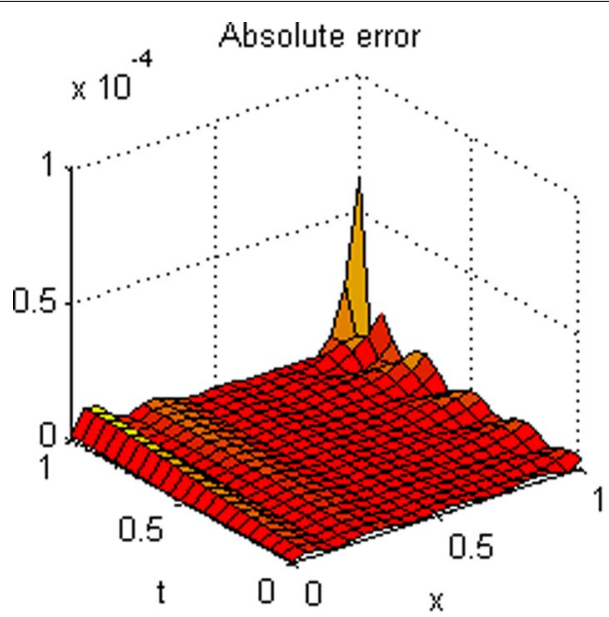

Fig. 7 Absolute error of Example 2 

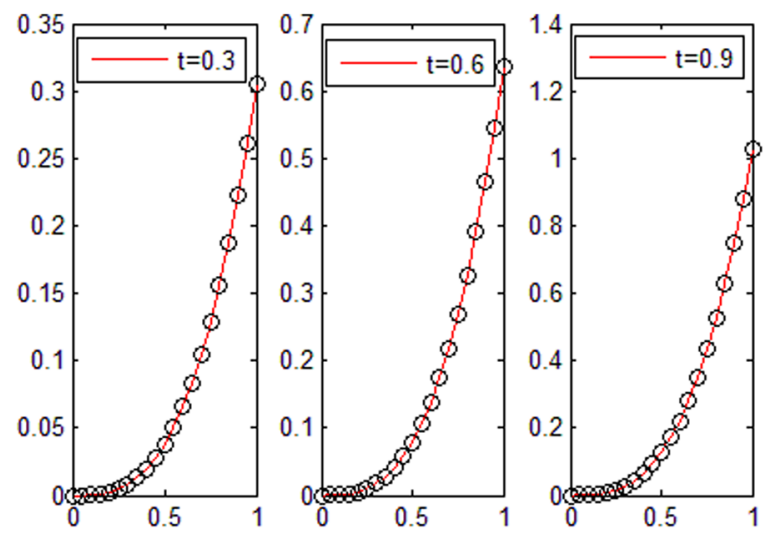

Fig. 8 Numerical and exact solution in different values of $t$ for Example 2

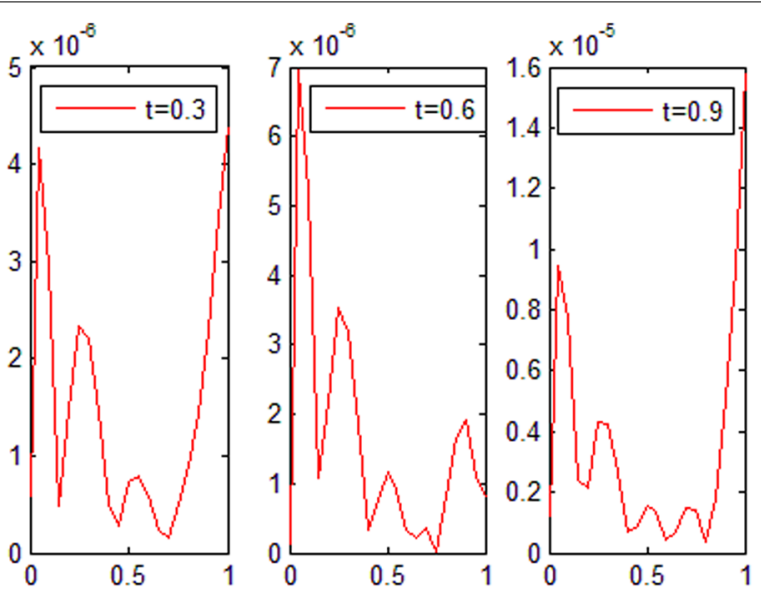

Fig. 9 Absolute error in different values of $t$ for Example 2

convergence precision for one-dimensional convection diffusion equations with variable coefficients. Table 1 and Fig. 11 show that the convergence and accuracy of the proposed algorithm is very good, with $M$ and $N$ increase. Moreover, a small $M$ and $N$ can achieve high precision.

Table 1 Absolute error between approximate and exact solutions at $\boldsymbol{t}=\mathbf{0 . 3}$ for Example 2

\begin{tabular}{lllll}
\hline $\mathbf{x}$ & Exact solution & $\boldsymbol{M}=\boldsymbol{N = \mathbf { 3 }}$ & $\mathbf{M = N = \mathbf { 5 }}$ & $\mathbf{M = N = \mathbf { 7 }}$ \\
\hline 0.1 & 0.0003 & $1.63 \mathrm{e}-005$ & $3.06 \mathrm{e}-006$ & $2.68 \mathrm{e}-006$ \\
0.2 & 0.0024 & $4.85 \mathrm{e}-005$ & $2.08 \mathrm{e}-006$ & $1.42 \mathrm{e}-006$ \\
0.3 & 0.0082 & $5.24 \mathrm{e}-005$ & $2.60 \mathrm{e}-007$ & $2.06 \mathrm{e}-006$ \\
0.4 & 0.0195 & $3.67 \mathrm{e}-005$ & $9.20 \mathrm{e}-007$ & $4.50 \mathrm{e}-007$ \\
0.5 & 0.0381 & $1.04 \mathrm{e}-005$ & $9.70 \mathrm{e}-007$ & $6.90 \mathrm{e}-007$ \\
0.6 & 0.0658 & $1.76 \mathrm{e}-005$ & $1.10 \mathrm{e}-007$ & $6.00 \mathrm{e}-007$ \\
0.7 & 0.1045 & $3.85 \mathrm{e}-005$ & $1.10 \mathrm{e}-006$ & $7.00 \mathrm{e}-008$ \\
0.8 & 0.1559 & $4.33 \mathrm{e}-005$ & $1.98 \mathrm{e}-006$ & $3.90 \mathrm{e}-007$ \\
0.9 & 0.2220 & $2.33 \mathrm{e}-005$ & $2.14 \mathrm{e}-006$ & $1.84 \mathrm{e}-006$ \\
\hline
\end{tabular}


Examples 2 and 3 show that the absolute error also can reaches to $10^{-6}$ for general one-dimensional fractional convection diffusion equations with variable coefficients. The two examples show that the proposed approach is very feasible and effective in solving fractional convection diffusion equations under real backgrounds.

Example 3 Consider the convection diffusion equations with variable coefficients

$$
b(x) \frac{\partial u}{\partial x}+\frac{\partial u}{\partial t}=a(x) \frac{\partial^{\alpha} u}{\partial x^{\alpha}}+g(x, t) ; \quad 0<x<1, t>0
$$

where $a(x)=\frac{\Gamma(2.2)}{6} x^{2.8}, \quad b(x)=\frac{x^{2}}{3} ;$ with $g(x, t)=-x^{3} e^{-t}$ and the boundary condition:

$$
\begin{aligned}
& u(x, 0)=x^{3} \\
& u(0, t)=0, \quad u(1, t)=e^{-t}, \quad t>0 .
\end{aligned}
$$

The exact solution of this problem for $\alpha=1.8$ is $u(x, t)=x^{3} e^{-t}$. The graphs of comparison between numerical and analytical solution for $M=N=6$ in some values of $t$ are shown in Fig. 10. The graphs of absolute error for different $M$ and $N$ in some values of $t$ are also shown in Fig. 11.

Example 4 Consider the convection diffusion Eq. (52), with $a(x)=\frac{x^{2}}{6}, b(x)=\frac{x}{3}$ $g(x, t)=-x^{3} e^{-t}$. The exact solution of this problem when $\alpha=2$ is $u(x, t)=x^{3} e^{-t}$. The values of exact solution $(\alpha=2)$ and approximate solution for some different values of $\alpha$ and some nodes $(x, t)$ in $(0,1) \times(0,1)$, when $M=N=3$ are shown in Table 2.

By comparing the data in Table 2, we can see the numerical solutions agree with the analytical solution $(\alpha=2)$ well with the fractional order gradually approximate to the order of $\alpha=2$. The example is introduced to verify the stability of the proposed algorithm.

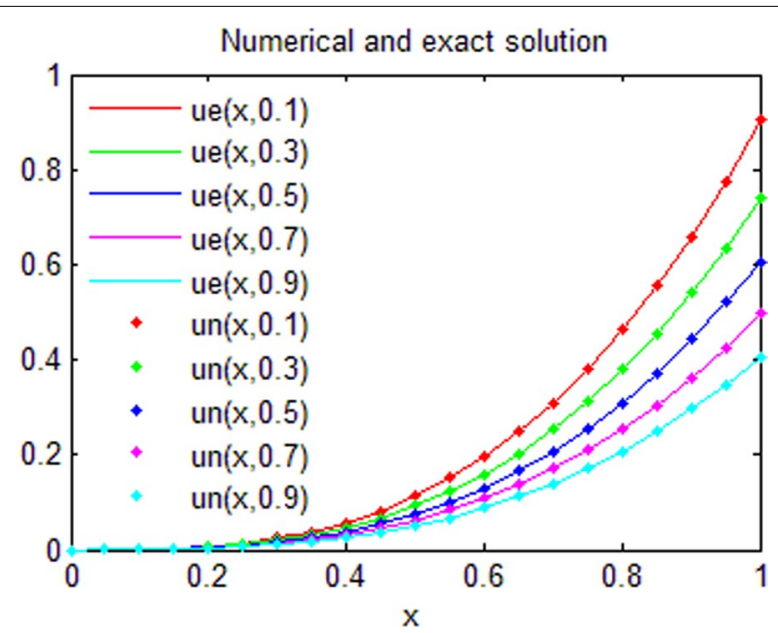

Fig. 10 Comparison between numerical and analytical solution for Example 3 

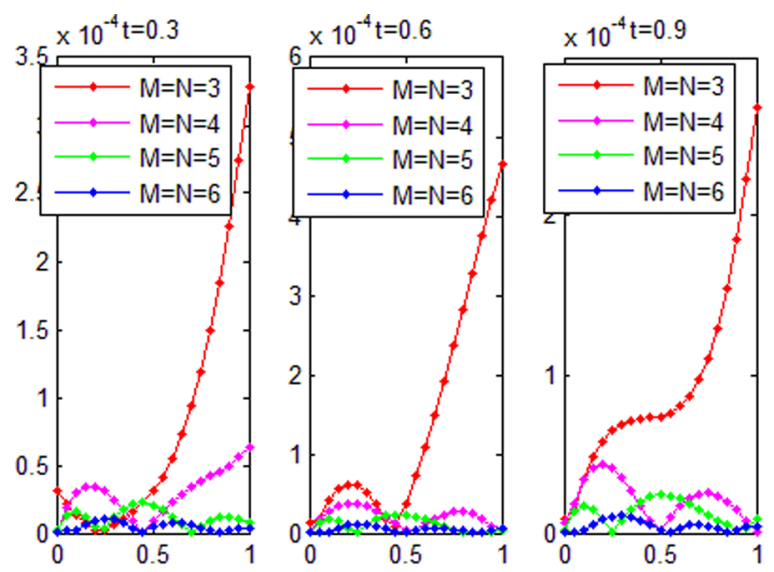

Fig. 11 Absolute error for different $M$ and $N$ in some times for Example 3

Table 2 Absolute error for different fractional order

\begin{tabular}{llllll}
\hline$(\boldsymbol{x}, \boldsymbol{t})$ & $\boldsymbol{a}=\mathbf{2}$ & $\boldsymbol{a}=\mathbf{1 . 9 5}$ & $\boldsymbol{a}=\mathbf{1 . 9}$ & $\boldsymbol{a}=\mathbf{1 . 8 5}$ & $\boldsymbol{a}=\mathbf{1 . 8}$ \\
\hline$(0.2,0.2)$ & $6.31 \mathrm{e}-05$ & 0.0011 & 0.0025 & 0.0044 & 0.0066 \\
$(0.4,0.4)$ & $7.45 \mathrm{e}-05$ & 0.0006 & 0.0010 & 0.0011 & 0.0010 \\
$(0.6,0.6)$ & $1.64 \mathrm{e}-04$ & 0.0034 & 0.0069 & 0.0109 & 0.0154 \\
$(0.8,0.8)$ & $5.81 \mathrm{e}-05$ & 0.0040 & 0.0085 & 0.0140 & 0.0205 \\
\hline
\end{tabular}

\section{Conclusion}

Here a new operational method to approximate the numerical solution of one-dimensional fractional convection diffusion equations have been introduced. To this end, the new operational matrix of fractional-order differentiation is obtained. It appears that using Chebyshev operational matrix algorithm will give more accurate solutions than other existing methods. The approach is computationally efficient and the algorithm can be implemented easily on a computer. The advantage of the methods is that only small size operational matrix is required to provide the solutions at high accuracy. Numerical examples are given to show that the proposed algorithm is robust, efficient and applicable.

\section{Authors' contributions}

JQX carried out the study. QXH and XY supervised the work. JQX proposed the method for the analysis and all authors conducted the analysis. JQX prepared the manuscript under the guidance of QXH and XY. All authors read and approved the final manuscript.

\section{Acknowledgements}

The work is supported by National Youth Foundation "Research on thermal-mechanical coupling elastoplastic fast multipole Boundary Element Method of metal plastic forming process" (51504157).

\section{Competing interests}

The authors declare that they have no competing interests. 


\section{References}

Abbasbandy S, Kazem S, Alhuthali MS (2015) Application of the operational matrix of fractional-order Legendre functions for solving the time-fractional convection-diffusion equation. Appl Math Comput 266:31-40

Bhrawy AH, Alofi AS, Ezz-Eldien SS (2011) A quadrature tau method for fractional differential equations with variable coefficients. Appl Math Lett 24(12):2146-2152

Bhrawy AH, Doha EH, Baleanu D (2015) A spectral tau algorithm based on Jacobi operational matrix for numerical solution of time fractional diffusion-wave equations. J Comput Phys 293:142-156

Calo VM, Chung ET, Efendiev Y (2015) Multiscale stabilization for convection-dominated diffusion in heterogeneous media. arXiv preprint: 1509.06833

Chen LL, Jin FJ (2007) A study on convection-diffusion Kinetics model in dyeing processes. http://www.paper.edu.cn/ releasepaper/content/200710-361

Chen YM, Wu YB, Cui YH (2010) Wavelet method for a class of fractional convection-diffusion equation with variable coefficients. J Comput Sci 1:146-149

Chen YM, Sun YN, Liu LQ (2014) Numerical solution of fractional partial differential equations with variable coefficients using generalized fractional-order Legendre functions. Appl Math Comput 244:847-858

Colla L, Fedele L, Buschmann MH (2015) Laminar mixed convection of $\mathrm{TiO}_{2}$-water nanofluid in horizontal uniformly heated pipe flow. Int J Therm Sci 97:26-40

Das $\mathrm{P}$ (2015) Comparison of a priori and a posteriori meshes for singularly perturbed nonlinear parameterized problems. J Comput Appl Math 290:16-25

Das P, Mehrmann V (2015) Numerical solution of singularly perturbed convection-diffusion-reaction problems with two small parameters. BIT Numer Math 56(1):1-26

Das P, Natesan S (2014) Optimal error estimate using mesh equidistribution technique for singularly perturbed system of reaction-diffusion boundary-value problems. Appl Math Comput 249:265-277

Dehghan M, Saadatmandi A (2006) A Tau method for the one-dimensional parabolic inverse problem subject to temperature over specification. Comput Math Appl 52(6-7):933-940

Diehl S (2015) Numerical identification of constitutive functions in scalar nonlinear convection-diffusion equations with application to batch sedimentation. Appl Numer Math 95:154-172

Doha EH, Bhrawy AH, Ezz-Eldien SS (2011) A Chebyshev spectral method based on operational matrix for initial and boundary value problems of fractional order. Comput Math Appl 62:2364-2373

El-Gamel M (2006) A Wavelet-Galerkin method for a singularly perturbed convection-dominated diffusion equation. Appl Math Comput 181:1635-1644

Fang J, Deng BL (2014) Rejection and modeling of arsenate by nanofiltration: contributions of convection, diffusion and electromigration to arsenic transport. J Membr Sci 453:42-51

Farahani A, Taghaddos H, Shekarchi M (2015) Prediction of long-term chloride diffusion in silica fume concrete in a marine environment. Cement Concrete Compos 59:10-17

He MY, Sun PT, Wang C (2015) A two-grid combined finite element-upwind finite volume method for a nonlinear convection-dominated diffusion reaction equation. J Comput Appl Math 288:223-232

Hernandez J, Crespo A, Duijm NJ (1995) Numerical modeling of turbulent jet diffusion flames in the atmospheric surface layer. Combust Flame 101(1-2):113-131

Hu GJ, Zhao L, Wu XD (2016) New Fourier-series-based analytical solution to the conduction-convection equations to calculate soil temperature, determine soil thermal properties, or estimate water flux. Int J Heat Mass Transf 95:815-823

Karalashvili M, Marquardt W, Mhamdi A (2015) Optimal experimental design for identification of transport coefficient models in convection-diffusion equations. Comput Chem Eng 80:101-113

Kaya A (2015) Finite difference approximations of multidimensional unsteady convection-diffusion-reaction equations. J Comput Phys 285:331-349

Li N, Zhao JP, Feng XL (2016) Generalized polynomial chaos for the convection diffusion equation with uncertainty. Int J Heat Mass TranSF 97:289-300

Liu L, Zheng LC, Liu FW (2016) Anomalous convection diffusion and wave coupling transport of cells on comb frame with fractional Cattaneo-Christov flux. Commun Nonlinear Sci Numer Simul 38:45-58

Mudunuru MK, Nakshatrala KB (2016) On enforcing maximum principles and achieving element-wise species balance for advection-diffusion-reaction equations under the finite element method. J Comput Phys 305:448-493

Su NH (2014) Mass-time and space-time fractional partial differential equations of water movement in soils: theoretical framework and application to infiltration. J Hydrol 519:1792-1803

Wu WJ, Feng XL, Liu DM (2013) The local discontinuous Galerkin finite element method for a class of convection-diffusion equations. Nonlinear Anal Real 14:734-752

Zaib A, Shafile S (2014) Thermal diffusion and diffusion thermo effects on unsteady MHD free convection flow over a stretching surface considering Joule heating and viscous dissipation with thermal stratification, chemical reaction and Hall current. J Frankl I 351:1268-1287

Zhou FY, Xu XY (2014) Numerical solution of the convection diffusion equations by the second kind Chebyshev wavelets. Appl Math Comput 247:353-367

Zhou FY, Xu XY (2016) The third kind Chebyshev wavelets collocation method for solving the time-fractional convection diffusion equations with variable coefficients. Appl Math Comput 280:11-29 